Check for updates

Cite this: RSC Adv., 2018, 8, 38538

\title{
An energy self-compensating phosphosilicate material applied to temperature sensors $\dagger$
}

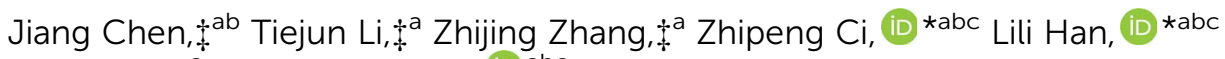 \\ Haiyan Jiao a and Yuhua Wang (D) abc
}

For years, researchers have been exploring effective methods of sustaining the emission intensity of phosphors with increasing temperature by suppressing emission loss. In this work, we developed a multi-cationic site and lattice-distorted phosphosilicate phosphor, $\mathrm{Ca}_{8} \mathrm{Al}_{2} \mathrm{P}_{6} \mathrm{SiO}_{28}$ : $\mathrm{Ce}$, Eu. To obtain luminous-self-healing properties, we attempted to change the energy depths and density distributions of the traps to achieve self-suppression of emission loss by energy compensation from the traps or energy transfer between $\mathrm{Ce}^{3+}$ and $\mathrm{Eu}^{2+} / \mathrm{Eu}^{3+}$. The temperature-dependent emission spectra indicate that the luminescence of $\mathrm{Ce}^{3+}$ presents similar change trends in both single and co-doped samples. Meanwhile, the change trends of the $\mathrm{Eu}^{2+} / \mathrm{Eu}^{3+}$ emission intensities show obvious differences. Combined with the thermoluminescence curves, decay times, temperature-dependent fluorescence characteristics and cathodoluminescence spectra, we speculate that the traps play an important role in the luminescence of $\mathrm{Ce}^{3+}$ due to the smaller energy difference of the $\mathrm{Ce}^{3+}$ excited states and the conduction band. The abnormal luminescence of $\mathrm{Eu}^{2+} / \mathrm{Eu}^{3+}$ mainly results from the energy transfer of $\mathrm{Ce}^{3+}$ to $\mathrm{Eu}^{2+} / \mathrm{Eu}^{3+}$. For this phenomenon, a high thermal sensitive fluorescence intensity ratio is obtained in a broad temperature range, which implies that this material can be applied in temperature sensors.

Received 11th September 2018 Accepted 28th October 2018

DOI: $10.1039 / c 8 \mathrm{ra0} 07566 \mathrm{~b}$

rsc.li/rsc-advances decreases the luminescence intensity when the phosphor is operated at elevated temperature. For years, researchers have been exploring effective methods to sustain the emission intensity of phosphors with increasing temperature by suppressing emission loss from non-radiative transitions by a counter mechanism. Recently, some beneficial results have been obtained. In a 2017 Nature Materials article, Yoon Hwa Kim et al. reported an interesting host that can absorb excess thermal energy, generate structural transformation, and then engender defect levels; these serve as electron-trapping centers, transferring energy to the assisted ions by thermal activation from traps. ${ }^{19}$ That is to say, appropriate traps are a key factor and ultimately play an important role in the process of suppressing emission loss. In view of this thought, we hoped to design a multi-cationic site material in which rational traps can be introduced by adjusting and optimizing the synthesis scheme to decrease the probability of energy loss. Combined with multi-activators, we expected to exploit the specific energy compensation from traps or energy transfer (ET) from the sensitizer to the activator to suppress or even tailor the energy loss of the luminescence centers in the heating process. In this paper, we selected the phosphosilicate $\mathrm{Ca}_{8} \mathrm{Al}_{2} \mathrm{P}_{6} \mathrm{SiO}_{28}$ (CAPS) as a general luminescent matrix. CAPS has a mineral whitlockite structure that is similar to that of $\beta-\mathrm{Ca}_{3}\left(\mathrm{PO}_{4}\right)_{2}$. In this structure, there are six different sites that can offer changeable crystal environments. Especially, these cationic sites are not completely filled, which is beneficial to produce traps with 
different depths. Additionally, we can also replace the partial $\left[\mathrm{PO}_{4}\right]^{3-}$ tetrahedra with $\left[\mathrm{SiO}_{4}\right]^{4-}$ tetrahedra to further adjust the energy and density distributions of the traps. A series of CAPS:Ce, Eu phosphors were successfully synthesized by solid state reactions at high temperature, and we carefully studied their structures, luminescence characteristics, trap distributions and detailed physical processes of electron transition with increasing temperature by XRD, photoluminescence (PL), photoluminescence excitation (PLE) spectra, decay times, temperature-dependent fluorescence studies, cathodoluminescence (CL) spectra, etc.

\section{Experimental section}

\subsection{Materials and synthesis}

The CAPS:0.03Ce, $x$ Eu samples were synthesized by high temperature solid-state reactions. Firstly, the raw materials $\mathrm{Eu}_{2} \mathrm{O}_{3}\left(4 \mathrm{~N}\right.$ ), $\mathrm{CaCO}_{3}$ (A.R.), $\mathrm{SiO}_{2}$ (A.R.), $\mathrm{Al}_{2} \mathrm{O}_{3}$ (A.R.), $\mathrm{CeO}_{2}$ (4 N) and $\left(\mathrm{NH}_{4}\right)_{2} \mathrm{HPO}_{4}$ (A.R.) were weighed in an agate mortar. The samples were then calcined in a crucible for 2 hours in air at $900{ }^{\circ} \mathrm{C}$ to release $\mathrm{NH}_{3}, \mathrm{CO}_{2}$ and $\mathrm{H}_{2} \mathrm{O}$ and were then heated for 6 hours in a fluidized $\mathrm{N}_{2}-\mathrm{H}_{2}(5 \%)$ reduction atmosphere at $1250{ }^{\circ} \mathrm{C}$ to $1300{ }^{\circ} \mathrm{C}$. Finally, the samples were cooled to room temperature and ground into powder for further characterization.

\subsection{Measurements and characterization}

The crystal structures of the samples were characterized by powder X-ray diffraction (Rigaku D/Max-2400 X-ray diffractometer) and XRD analysis with CuK $\alpha$ rays filtered by nickel. The PL, PLE and attenuation curves of the samples were measured using a $450 \mathrm{~W}$ Xe light source and a ns pulse hydrogen lamp on a FLS-920T fluorescence spectrophotometer (Livingston, UK). All measurements were carried out at room temperature. The thermal quenching was tested by a heating device (TAP-02) combined with PL equipment. The TL curves were measured by an FJ-417A TL measuring instrument (Beijing nuclear instrument plant, Beijing, China). Low-voltage CL spectra were obtained using a modified Mp-Micro-S instrument.

\section{Results and discussion}

\subsection{Phase identification}

The representative XRD diagrams of single and co-doped CAPS samples with $\mathrm{Ce}$ and $\mathrm{Eu}$ ions are given in Fig. S1† and 1. The diffraction peaks of all the samples can be attributed to the pure phase of $\mathrm{Ca}_{9} \mathrm{AlP}_{7} \mathrm{O}_{28}$ (CAP) by its standard JCPDS card (no. 481192). No other phases were detected, indicating that the samples were single phase. CAP is related to the mineral pyroxene and has the same structure as $\beta-\mathrm{Ca}_{3}\left(\mathrm{PO}_{4}\right)_{2}$. In the host, there are six cation sites: M1 to M6. The M1, M2, and M3 sites are eight-coordinated with oxygen atoms and are fully occupied by $\mathrm{Ca}^{2+}$. The M4 site is surrounded by nine oxygen atoms and is $50 \%$ occupied by $\mathrm{Ca}^{2+}$. The distorted octahedral M5 site is fully occupied by $\mathrm{Ca}^{2+}$. The M6 site is vacant. In this experiment, the diffraction peaks of the CAPS:0.03Ce and $x$ Eu samples shifted to

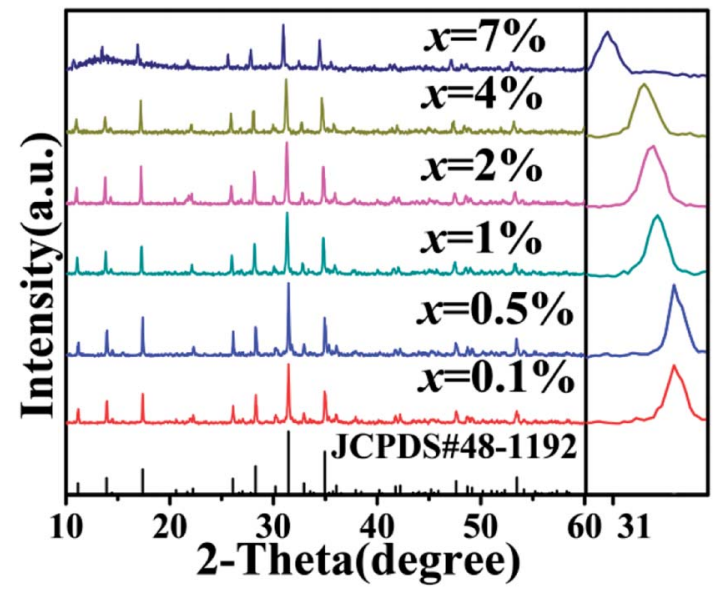

Fig. 1 The XRD patterns of CAP and the CAPS:0.03Ce, $x$ Eu samples ( $x$ $=0.1 \%, 0.5 \%, 1 \%, 2 \%, 4 \%, 7 \%$ ).

smaller angles with increasing doping ion concentration. In the reference crystal radii of all the species, ${ }^{20}$ the effective ionic radii for eight-coordinated $\mathrm{Ca}^{2+}, \mathrm{Ce}^{3+}, \mathrm{Eu}^{2+}$, and $\mathrm{Eu}^{3+}$ are 1.12, 1.143, 1.25 and $1.066 \AA$ A, respectively. For nine-coordinated $\mathrm{Ca}^{2+}, \mathrm{Ce}^{3+}$, $\mathrm{Eu}^{2+}$, and $\mathrm{Eu}^{3+}$, the effective ionic radii are 1.18, 1.196, 1.3 and $1.12 \AA$, respectively. For six-coordinated $\mathrm{Al}^{3+}, \mathrm{Ce}^{3+}, \mathrm{Eu}^{2+}$, and $\mathrm{Eu}^{3+}$, the effective ionic radii are $0.39,1.01,1.17$ and $0.947 \AA$, respectively. It is obvious that the shifts are caused by the differences in the ionic radii. According to a report by B. I. Lazoryak et al., ${ }^{21}$ solid solutions in $\mathrm{Ca}_{9} \mathrm{RP}_{7} \mathrm{O}_{28}$ systems can be formed in this region for all trivalent elements with radii $0.5 \leq r$ $\leq 1.3 \AA$ A. Large cations, such as some rare earth elements, prefer to occupy the M1 to M3 positions, while small cations ( $\mathrm{Al}^{3+}$, $\mathrm{Fe}^{3+}$, etc.) prefer to occupy the M5 position. Thus, we consider that the doped ions $\mathrm{Ce}^{3+}, \mathrm{Eu}^{2+}$, and $\mathrm{Eu}^{3+}$ are likely to occupy the four sites M1 to M4; this speculation is further proved in the following spectrum analysis. The structure diagram of CAPS is shown in Fig. S2. $\dagger$

\subsection{Photoluminescence property analysis}

Fig. 2 shows the PLE spectrum monitored at $339 \mathrm{~nm}$ and the PL spectrum excited at $296 \mathrm{~nm}$ of CAPS: $0.03 \mathrm{Ce}^{3+}$; the PLE spectrum (black line) contains three obvious absorption bands at 265, 296 and $313 \mathrm{~nm}$ due to the $4 \mathrm{f}-5 \mathrm{~d}$ transition of $\mathrm{Ce}^{3+}$, with the highest peak at $296 \mathrm{~nm}$. Upon excitation at $296 \mathrm{~nm}$, CAPS:0.03Ce $\mathrm{Ce}^{3+}$ exhibits a broad purple-blue emission band in the range from 310 to $420 \mathrm{~nm}$ that peaks at $339 \mathrm{~nm}$. To further determine the crystallographic sites of the doping ions in the CAPS host, the coordination number $n$ can be calculated by the following empirical relation: ${ }^{22}$

$$
\begin{gathered}
E=Q\left[1-\left(\frac{V}{4}\right)^{\frac{1}{V}} \times 10^{-n \times \mathrm{ea} \times \frac{r}{80}}\right] \\
n=-80 \lg \left\{[1-E / Q] /(V / 4)^{\frac{1}{V}}\right\} /(\text { ea }) r
\end{gathered}
$$




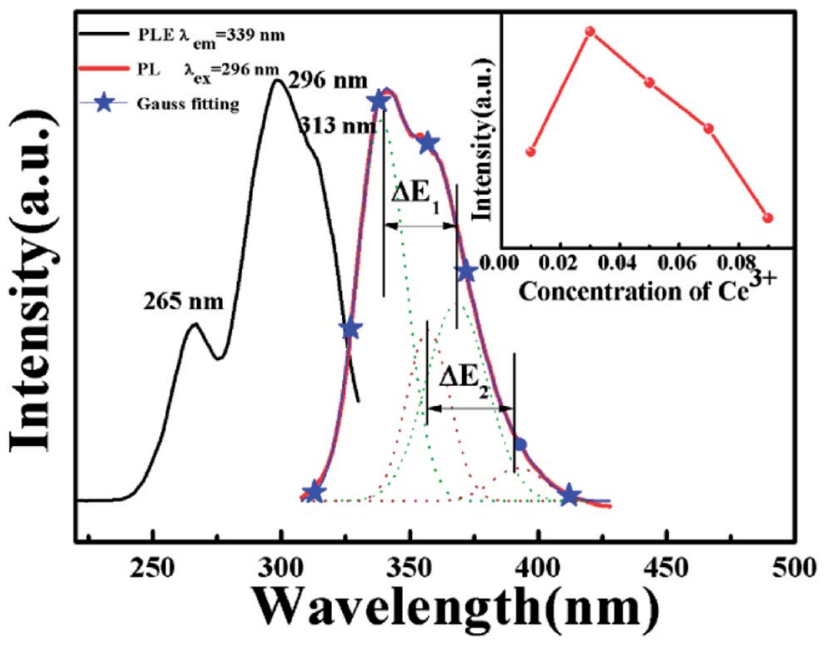

Fig. 2 PLE spectrum monitored at $339 \mathrm{~nm}$ and PL spectrum excited at $296 \mathrm{~nm}$ of CAPS: $0.03 \mathrm{Ce}^{3+}$; the inset shows the curve of the intensity of $\mathrm{Ce}^{3+}$ with concentration at $339 \mathrm{~nm}$.

where $Q$ is the lowest energy position at the edge of the $\mathrm{d}$ band of the free ion (here, $Q=50000 \mathrm{~cm}^{-1}$ for $\mathrm{Ce}^{3+}$ ), $V$ is the valence state of the active ion, $n$ is the coordination number, ea is the electron affinity of the anion, and $r$ is the radius of the cation $\left(\mathrm{Ca}^{2+}\right)$ replaced by the active cation. The value of the parameter $n$ was calculated to be 8 to 10 . That is, the doping ion $\mathrm{Ce}^{3+}$ may replace two different eight-coordinated sites (M1, M2, M3) and one nine-coordinated site (M4), which corresponds with the XRD measurement results. Because the crystal environments of M1, M2 and M3 are very similar, the emission bands of the active ions in the three sites are difficult to distinguish. In order to simplify the analysis, we assume that the active ions in the M1, M2, and M3 sites only show emission bands. For the sample CAPS: $0.03 \mathrm{Ce}^{3+}$, the emission spectrum in Fig. 2 can be readily decomposed into four separate Gaussian components centered at $340 \mathrm{~nm}\left(29412 \mathrm{~cm}^{-1}\right), 365 \mathrm{~nm}\left(27392 \mathrm{~cm}^{-1}\right)$, $359 \mathrm{~nm}\left(27855 \mathrm{~cm}^{-1}\right)$ and $390 \mathrm{~nm}\left(25641 \mathrm{~cm}^{-1}\right)$ by the Gauss curve fitting. The energy difference $\Delta E_{1}$ between 340 and $365 \mathrm{~nm}$ is $2020 \mathrm{~cm}^{-1}$ and $\Delta E_{2}$ between 359 and $390 \mathrm{~nm}$ is $2214 \mathrm{~cm}^{-1}$, which is very close to the usual energy difference between the $\mathrm{Ce}^{3+}{ }^{3+} \mathrm{F}_{\mathrm{J}}(J=7 / 2,5 / 2)$ states. ${ }^{23}$ From eqn (1), we can see that the larger the value of $n \times r$, the higher the value of $E$. Thus, the fitting peaks at 340 and $365 \mathrm{~nm}$ and 359 and $390 \mathrm{~nm}$ can be attributed to the nine- and eight-coordinated sites, respectively. In addition, the inset of Fig. 2 shows the curve of concentration quenching of $\mathrm{Ce}^{3+}$ and the optimal emission intensity located at $x=0.03$.

Fig. 3 shows the PLE and PL spectra of the phosphor CAP$\mathrm{S}: 0.03 \mathrm{Eu}$. The excitation spectrum was monitored at $614 \mathrm{~nm}$, and the corresponding excitation spectrum includes broad absorption bands and some line absorptions. The charge transfer band (CTB) of $\mathrm{O}^{2}-\mathrm{Eu}^{3+}$ has the largest broadband absorption at $260 \mathrm{~nm}^{24-27}$ The strong sharp lines at 394 and $465 \mathrm{~nm}$ correspond to the characteristic $\mathrm{f}-\mathrm{f}$ transitions of $\mathrm{Eu}^{3+}$. When monitored at $451 \mathrm{~nm}$, the corresponding PLE spectrum simply consists of a strong emission broadband that peaks at

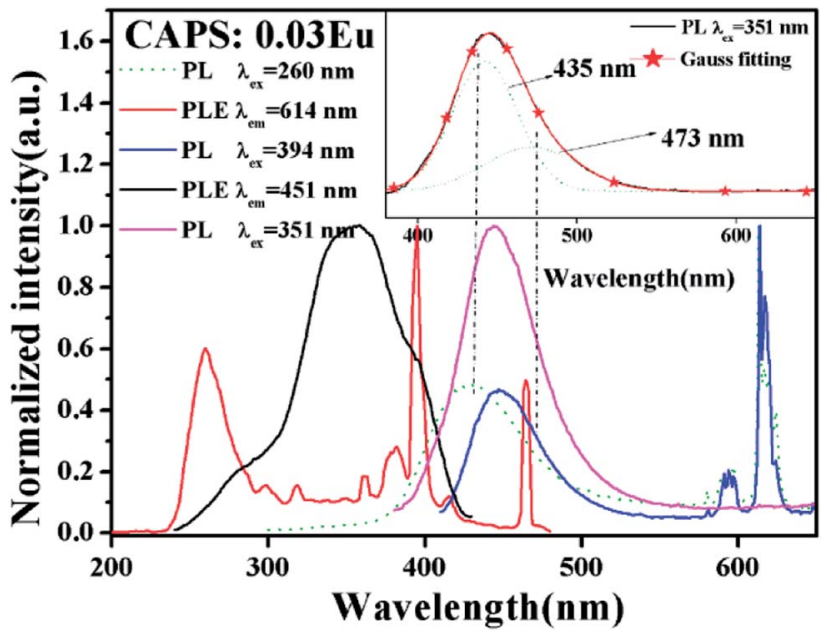

Fig. 3 The PLE spectra monitored at 451 and $614 \mathrm{~nm}$ and the PL spectra excited at 260,351, and $394 \mathrm{~nm}$ of CAPS:0.03Eu. The inset shows the Gauss curves of the PL spectra of CAPS:0.03Eu under excitation at $351 \mathrm{~nm}$.

$351 \mathrm{~nm}$; this results from the $4 \mathrm{f}-5 \mathrm{~d}$ transition absorption of $\mathrm{Eu}^{2+}$. When comparing the black solid line with the red line, we can see that there is no $\mathrm{Eu}^{2+}$ broadband absorption when monitored at $614 \mathrm{~nm}$ (the red line). This indicates that there is no ET between $\mathrm{Eu}^{2+}$ and $\mathrm{Eu}^{3+}$; that is, the luminescence properties of $\mathrm{Eu}^{2+}$ and $\mathrm{Eu}^{3+}$ can be considered to be completely independent. Upon $260 \mathrm{~nm}$ excitation, the corresponding PL spectrum contains a broad band centered at $\sim 445 \mathrm{~nm}$ and some line peaks due to the $\mathrm{Eu}^{2+} \mathrm{d}-\mathrm{f}$ transition and $\mathrm{Eu}^{3+}$ characteristic $\mathrm{f}-\mathrm{f}$ transition, which suggests that $\mathrm{Eu}^{3+}$ has not been fully reduced under a flowing $\mathrm{N}_{2}-\mathrm{H}_{2}(5 \%)$ atmosphere in the host CAPS. The weak peak is located at about $593 \mathrm{~nm}$ and belongs to the ${ }^{5} \mathrm{D}_{0} \rightarrow{ }^{7} \mathrm{~F}_{1}$ magnetic dipole transition of $\mathrm{Eu}^{3+}$, which is insensitive to the site symmetry. The main peak at $614 \mathrm{~nm}$ is attributed to the ${ }^{5} \mathrm{D}_{0} \rightarrow{ }^{7} \mathrm{~F}_{2}$ electric dipole transition of $\mathrm{Eu}^{3+}$, indicating that $\mathrm{Eu}^{3+}$ occupies the asymmetric center of the main lattice. Upon $394 \mathrm{~nm}$ excitation, the PL spectrum shape is similar to that upon $260 \mathrm{~nm}$ excitation except for the broadband emission position and the slight difference in the full-width at haft-maximum of $\mathrm{Eu}^{2+}$, which may originate from the selective excitation of $\mathrm{Eu}^{2+}$ at the different sites. When excited at $351 \mathrm{~nm}$, the PL spectrum of CAPS:0.03Eu only shows an asymmetric broad band from 380 to $650 \mathrm{~nm}$ with a maximum wavelength at about $451 \mathrm{~nm}$, as shown in the inset of Fig. 3. By eqn (1) and (2) $\left(Q=3400 \mathrm{~cm}^{-1}\right)$, the $n$ for $\mathrm{Eu}^{2+}$ can be also calculated as 8 to 10 , which is similar to the case of $\mathrm{Ce}^{3+}$. By the Gaussian fitting, the asymmetric emission band can be fitted by two components with peaks at 435 and $473 \mathrm{~nm}$, respectively, ${ }^{28,29}$ which can be attributed to $\mathrm{Eu}^{2+}$ occupying two different sites with nine and eight-coordination.

Comparing the PLE spectra of $\mathrm{Eu}^{2+}, \mathrm{Eu}^{3+}$ with the PL spectrum of $\mathrm{Ce}^{3+}$ in Fig. 4(a), we can clearly see an obvious spectrum overlap in the range of 310 to $420 \mathrm{~nm}$; this implies that ET can occur between $\mathrm{Ce}^{3+}$ and $\mathrm{Eu}^{2+}, \mathrm{Eu}^{3+}$. In order to confirm the existence of ET, we measured the PLE spectra of CAPS:0.03Ce 

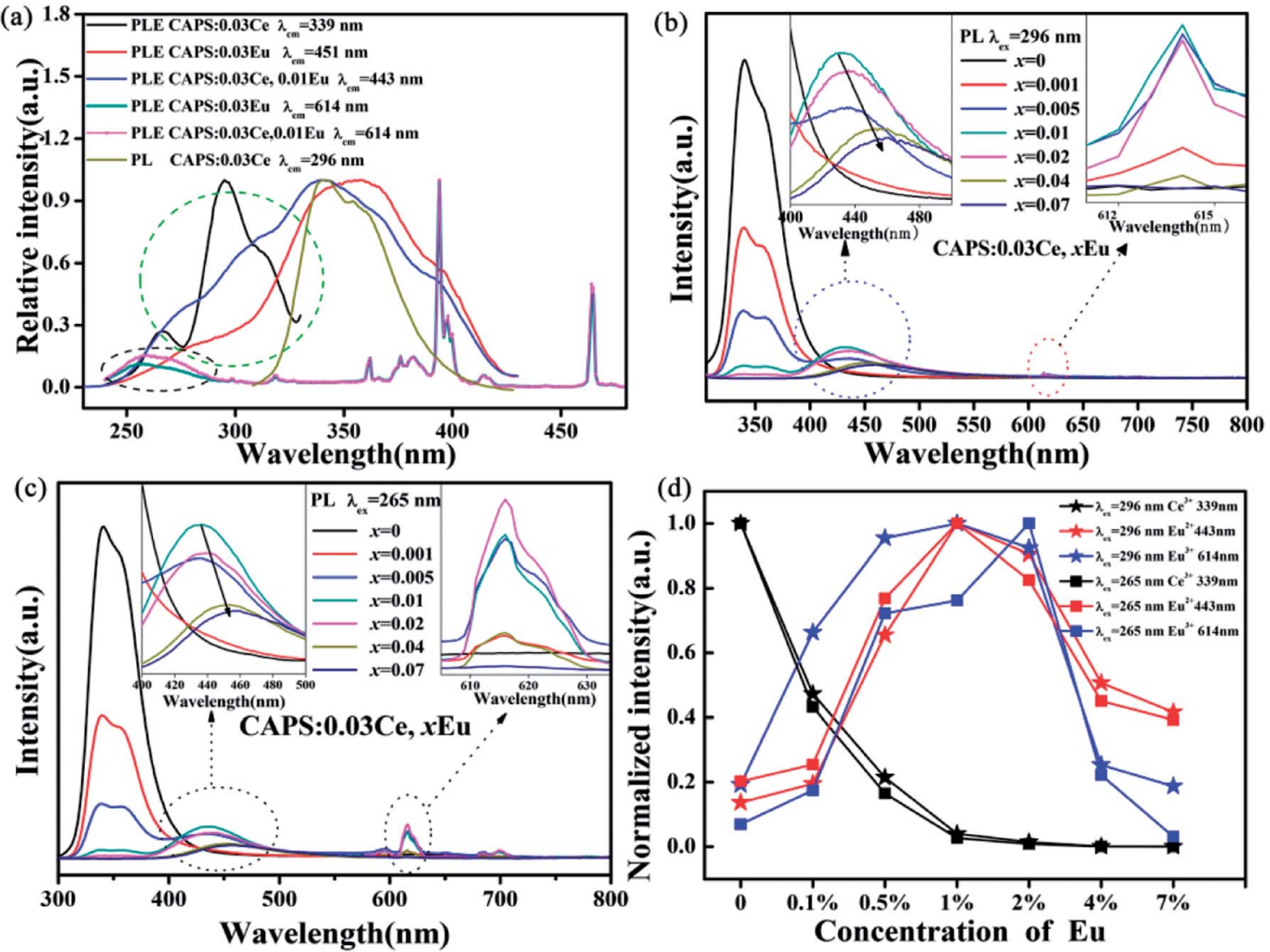

Fig. 4 (a) The PL spectra of CAPS:0.03Ce excited at $296 \mathrm{~nm}$ and the PLE spectra of CAPS:0.03Ce monitored at 339 nm, CAPS:0.03Ce, 0.01Eu monitored at 443 and $614 \mathrm{~nm}$ and CAPS:0.03Eu monitored at 451 and $614 \mathrm{~nm}$. (b) and (c) PL spectra of CAPS:0.03Ce, xEu excited at 296 and $265 \mathrm{~nm}$. (d) The emission intensities of Ce and Eu ions in CAPS:0.03Ce, $x$ Eu excited at 296 and $265 \mathrm{~nm}(0 \leq x \leq 0.07)$.

monitored at $339 \mathrm{~nm}$, CAPS:0.03Ce, 0.01Eu monitored at 443 and $614 \mathrm{~nm}$ and CAPS:0.03Eu monitored at 451 and $614 \mathrm{~nm}$. Compared to the PLE spectra of CAPS:0.03Eu monitored at 451 and $614 \mathrm{~nm}$, the PLE spectrum of CAPS:0.03Ce, 0.01Eu clearly has a new absorption band at about $296 \mathrm{~nm}$, marked by a green ellipse line, and a slight enhancement at about $265 \mathrm{~nm}$, marked by a black ellipse line. These phenomena indicate that the absorption of $\mathrm{Ce}^{3+}$ contributes to the emissions of $\mathrm{Eu}^{2+}$ and $\mathrm{Eu}^{3+}$ and thus proves that ET occurs between $\mathrm{Ce}^{3+}$ and $\mathrm{Eu}^{2+}$, $\mathrm{Eu}^{3+}$. Similar to the PLE spectrum of the Eu single-doped sample, the excitation spectrum of the $\mathrm{Ce}$, Eu co-doped sample monitored at $614 \mathrm{~nm}$ also does not present the obvious excitation of $\mathrm{Eu}^{2+}$, which indicates that ET does not similarly occur between $\mathrm{Eu}^{2+}$ and $\mathrm{Eu}^{3+}$ in CAPS:0.03Ce, 0.01Eu. Fig. 4(b) and (c) reveal the PL spectra of CAPS:0.03Ce, $x \mathrm{Eu}(0 \leq x$ $\leq 0.07$ ). Under excitation at 296 and $265 \mathrm{~nm}$, the PL spectra of the CAPS:0.03Ce, $x$ Eu samples consist of two emission bands due to $\mathrm{Ce}^{3+}$ (310 to 420 ) and $\mathrm{Eu}^{2+}$ (370 to 550 ) and several weak emission peaks of $\mathrm{Eu}^{3+}$ (above $575 \mathrm{~nm}$ ). As the Eu ion concentration $x$ increases, the emission intensity of the $\mathrm{Ce}^{3+}$ ions decreases monotonically, whereas the emission intensities of $\mathrm{Eu}^{2+}$ and $\mathrm{Eu}^{3+}$ simultaneously increase, as shown in the insets of Fig. 4(b) and (c); this also indirectly supports the occurrence of ET among $\mathrm{Ce}^{3+}, \mathrm{Eu}^{2+}$ and $\mathrm{Eu}^{3+} .{ }^{30}$ Meanwhile, with increasing $x$, the emission band of $\mathrm{Eu}^{2+}$ still shows a slight red shift, which is probably due to two reasons: the change in the crystal field environment and the selective excitation of $\mathrm{Eu}^{2+}$ at the different sites. In addition, as the Eu content increases, the distance between the sensitizer $\mathrm{Ce}^{3+}$ and the activators $\mathrm{Eu}^{2+}$ and $\mathrm{Eu}^{3+}$ becomes shorter. When the distances of $\mathrm{Ce}^{3+}, \mathrm{Eu}^{2+}$ and $\mathrm{Eu}^{3+}$ exceed the critical distances, concentration quenching will occur, as shown in Fig. 4(d). Upon $296 \mathrm{~nm}$ excitation, the optimal concentrations of both $\mathrm{Eu}^{2+}$ and $\mathrm{Eu}^{3+}$ are $1 \%$. However, under excitation at $265 \mathrm{~nm}$, the $\mathrm{Eu}^{3+}$ emission intensities achieve the maximum at $2 \%$. This may be due to the differences in ET efficiency between $\mathrm{Ce}^{3+}$ at different sites and $\mathrm{Eu}^{2+}$ and $\mathrm{Eu}^{3+}$ at different sites.

In order to more clearly understand the ET process, the lifetimes as well as the ET efficiencies were measured and calculated. Fig. 5(a) shows the decay curves of $\mathrm{Ce}^{3+}$ in CAPS:0.03Ce, $x$ Eu excited at $296 \mathrm{~nm}$ and monitored at $339 \mathrm{~nm}$. The corresponding luminescence decay times can be well fitted with a second-order exponential decay mode by the following equation: ${ }^{31}$

$$
I=A_{1} \exp \left(-t / \tau_{1}\right)+A_{2} \exp \left(-t / \tau_{2}\right)
$$

where $I$ is the luminescence intensity; $A_{1}$ and $A_{2}$ are the constants; $t$ is time; and $\tau_{1}$ and $\tau_{2}$ are the rapid and slow 
(a)

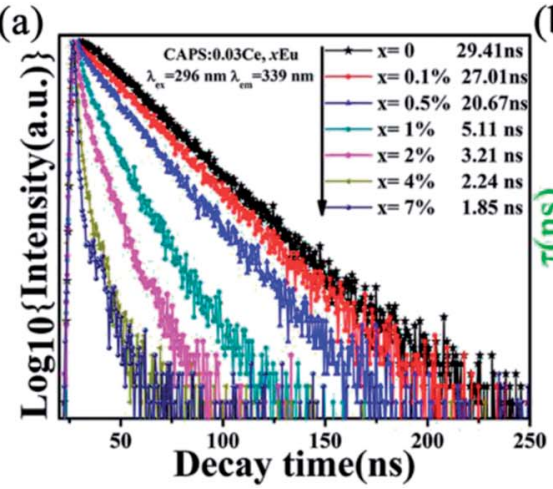

(b)

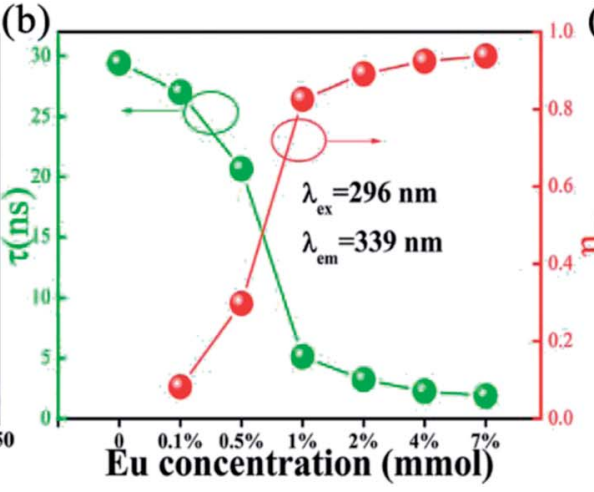

(c)

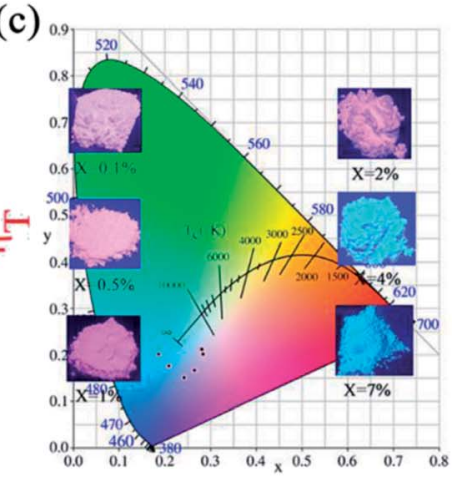

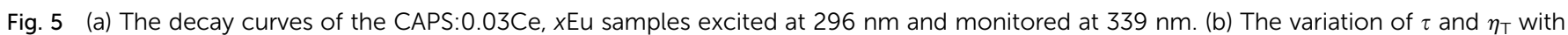

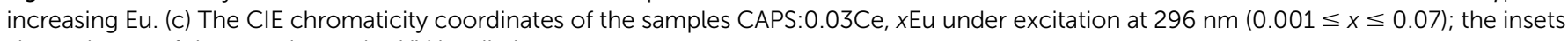
show photos of the samples under UV irradiation.

lifetimes for the exponential components, respectively. Using these parameters, the average decay times $(\tau)$ can be determined by the formula given below: ${ }^{32}$

$$
\tau=\left(A_{1} \tau_{12}+A_{2} \tau_{22}\right) /\left(A_{1} \tau_{1}+A_{2} \tau_{2}\right)
$$

The average decay times $(\tau)$ were calculated to be 27.01, 20.67, 5.11, 3.21, 2.24 and 1.85 ns for CAPS:0.03Ce, $x$ Eu with $x=$ $0,0.001,0.005,0.01,0.02,0.04$ and 0.07 , respectively. In this Ce and $\mathrm{Eu}$ ions co-doped system, the average decay times were found to shorten with increasing Eu content. The changing trend is also shown in Fig. 5(b), which strongly supports the ET process from $\mathrm{Ce}^{3+}$ to $\mathrm{Eu}^{2+}$ or $\mathrm{Eu}^{3+}$; this ET process may happen via a resonant-type mechanism. ${ }^{33}$

The efficiency of ET $\left(\eta_{\mathrm{T}}\right)$ from $\mathrm{Ce}^{3+}$ to $\mathrm{Eu}^{2+} / \mathrm{Eu}^{3+}$ in the CAPS:0.03Ce, $x \mathrm{Eu}$ sample was calculated using the following formula: ${ }^{34}$

$$
\eta_{\mathrm{T}}=1-\frac{\tau_{\mathrm{S}}}{\tau_{\mathrm{S}_{0}}}
$$

where $\tau_{\mathbf{S}_{0}}$ and $\tau_{\mathbf{S}}$ are the decay lifetimes of $\mathrm{Ce}^{3+}$ in the absence and presence of $\mathrm{Eu}^{2+}$ and $\mathrm{Eu}^{3+}$, respectively. The ET efficiency curve is shown in Fig. 5(b). In addition, the CIE chromaticity coordinates of the samples CAPS:0.03Ce, $x \mathrm{Eu}$ under excitation at $296 \mathrm{~nm}(0.001 \leq x \leq 0.07)$ and photos of the samples under UV irradiation are shown in Fig. 5(c); the emission color changes from pink to blue-green with increasing Eu ion content from 0 to 0.07 .

\subsection{Temperature-dependent photoluminescence behavior}

A comprehensive understanding of the temperature dependence of phosphors in the process of their application is indispensable due to the thermal problems in many running devices and fluorescent temperature sensors. ${ }^{35}$ Numerous investigations have discussed these thermal quenching behaviors. Two competing factors prevail:one is the activation energy of non-radiative relaxation, and the other is the photon and phonon coupling effect caused by temperature, which improves the luminescence intensity of the activator. The temperature- dependent luminescence properties for the CAPS:Ce, CAPS:Eu and CAPS:0.03Ce, 0.005Eu phosphors are traced in the temperature range from $25{ }^{\circ} \mathrm{C}$ to $250{ }^{\circ} \mathrm{C}$, and the results are shown in Fig. 6(a-c) . For the CAPS:Ce and CAPS:Eu phosphors, with increasing temperature, the emission intensities of all the samples gradually decline. The emission intensities of $\mathrm{Ce}^{3+}$, $\mathrm{Eu}^{2+}$ and $\mathrm{Eu}^{3+}$ at $250{ }^{\circ} \mathrm{C}$ are $81 \%, 10 \%$ and $47 \%$ of their initial intensities at $25{ }^{\circ} \mathrm{C}$, respectively. When $\mathrm{Ce}$ and $\mathrm{Eu}$ ions are codoped into the CAPS host, the decreasing trend of the $\mathrm{Ce}^{3+} \mathrm{PL}$ intensity does not change greatly, as shown in Fig. 6(d). As the PL intensity decline of $\mathrm{Eu}^{2+}$ becomes slower, the PL intensity of $\mathrm{Eu}^{3+}$ actually increases. The intensities of $\mathrm{Ce}^{3+}, \mathrm{Eu}^{2+}$ and $\mathrm{Eu}^{3+}$ in the sample CAPS:0.03 $\mathrm{Ce}^{3+}, 0.005\left(\mathrm{Eu}^{2+} / \mathrm{Eu}^{3+}\right)$ at $250{ }^{\circ} \mathrm{C}$ are $76 \%$, $58 \%$ and $152 \%$ of those at $25{ }^{\circ} \mathrm{C}$, respectively. In other words, $\mathrm{Eu}^{2+}$ and $\mathrm{Eu}^{3+}$ in the $\mathrm{Ce}, \mathrm{Eu}$ co-doped samples show good thermal stability, which can be easily explained by the ET from $\mathrm{Ce}^{3+}$ to $\mathrm{Eu}^{2+}, \mathrm{Eu}^{3+}$ under the stronger electron-phonon coupling with increasing temperature. Also, according to this speculation, the emission intensity of $\mathrm{Ce}^{3+}$ in the co-doped sample should rapidly decrease due to a large number of transferred electrons in the $\mathrm{Ce}^{3+}$ excited states to the $\mathrm{Eu}^{2+}, \mathrm{Eu}^{3+}$ excited states. However, the actual experimental data indicate that the $\mathrm{Ce}^{3+}$ luminescence demonstrates similarly excellent thermal stability in the two samples. Thus, there should be an additional physical process to compensate the loss of $\mathrm{Ce}^{3+}$. The traps are the only possible source of this additional physical process. In the following paragraphs, we will carefully analyse the effect of the traps.

In the previous structural analysis, we found that our CAPS samples have six cation sites. The M1, M2, M3 and M5 sites are fully occupied by $\mathrm{Ca}^{2+}$. The M4 site is $50 \%$ occupied by $\mathrm{Ca}^{2+}$. The M6 site is vacant. Therefore, when doping ions are introduced, especially in the case of non-equivalent replacement, cation vacancies are generated easily. Meanwhile, for the phosphate material, there are many reports about the occurrence of oxygen vacancies through oxygen escaping from sites during the sintering process under both reducing and air atmospheres. ${ }^{36}$ As a result, numerous traps may exist to capture electrons or holes in the host. Fig. 7(a) shows the thermoluminescence (TL) curves of the CAPS, CAPS:0.03Ce $\mathrm{Ce}^{3+}$, CAPS:0.03Eu, and CAPS:0.03Ce, 
(a) $\quad \begin{array}{rrrrr}50 & 100 & 150 & 200 & 250\end{array}$

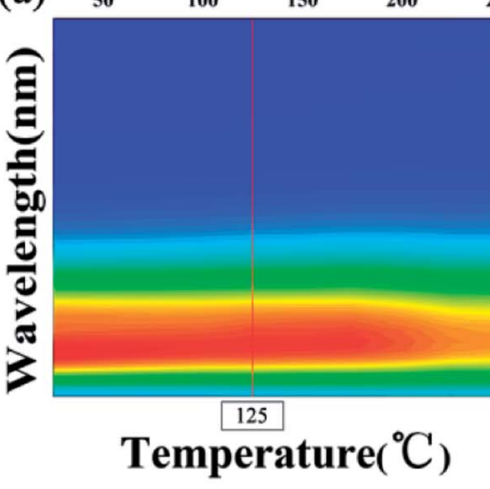

(c)
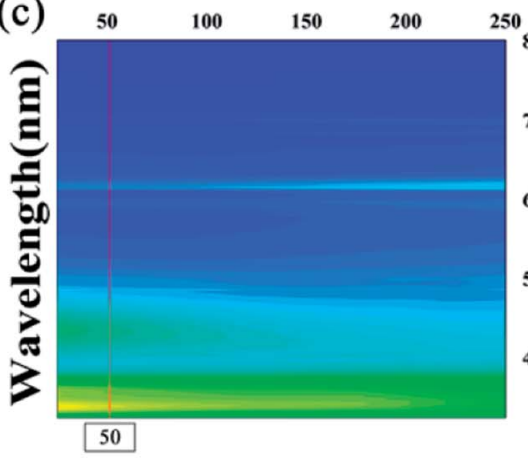

Temperature $\left({ }^{\circ} \mathrm{C}\right)$ (b)
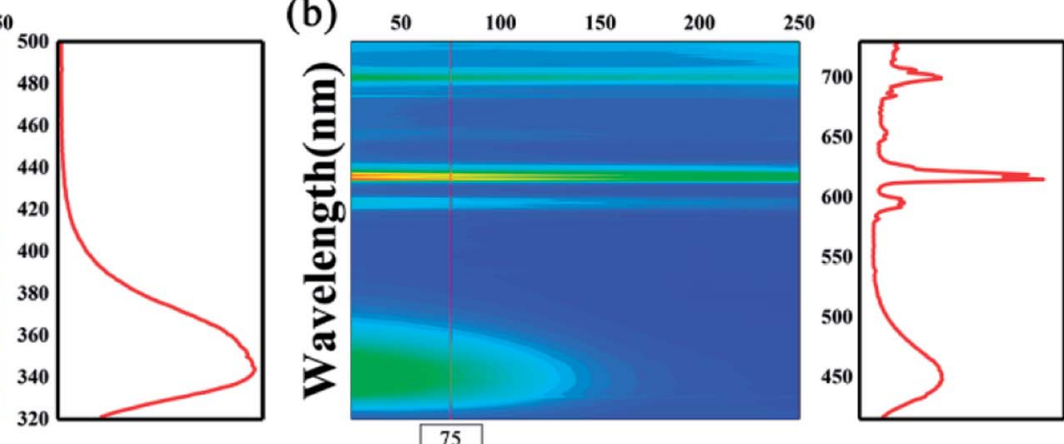

Temperature $\left({ }^{\circ} \mathrm{C}\right.$ )

(d)
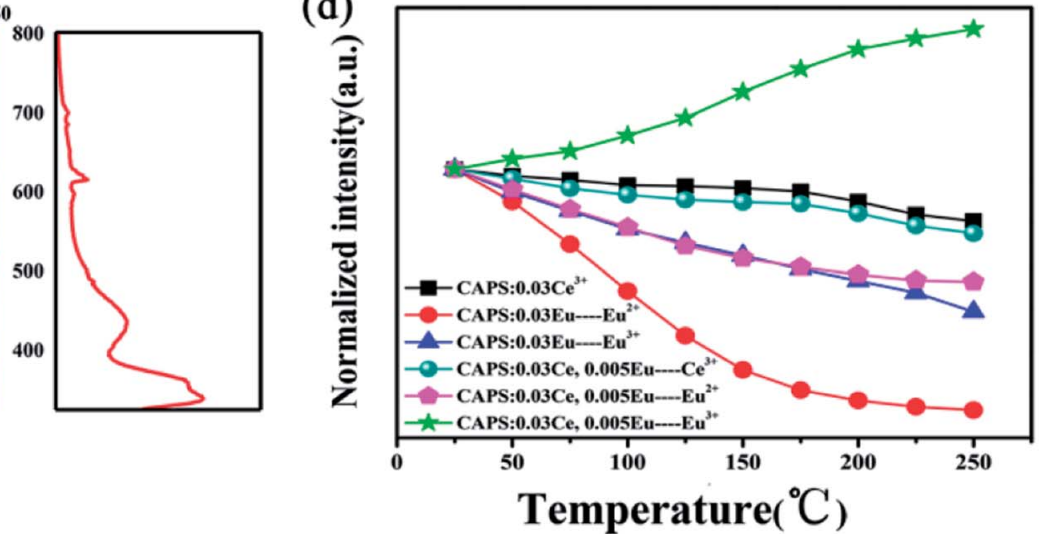

Fig. 6 The PL spectra of typical samples: (a) CAPS:0.03Ce, (b) CAPS:0.03Eu, and (c) CAPS:0.03Ce, 0.005Eu at various temperatures. (d) The dependence of the normalized PL intensities on temperature for typical phosphors.

$0.005 \mathrm{Eu}$ samples under the same conditions of exposure to preexcitation at $254 \mathrm{~nm}$ for $5 \mathrm{~min}$ before measurement. The heating rate of TL measurements is $1 \mathrm{~K} \mathrm{~s}^{-1}$. It can be seen that the
TL curve of the $\mathrm{Ce}^{3+}$-doped sample is similar to that of the host. Both samples have two broad bands; with the introduction of $\mathrm{Ce}^{3+}$, the bands slightly shift to higher temperatures. For the
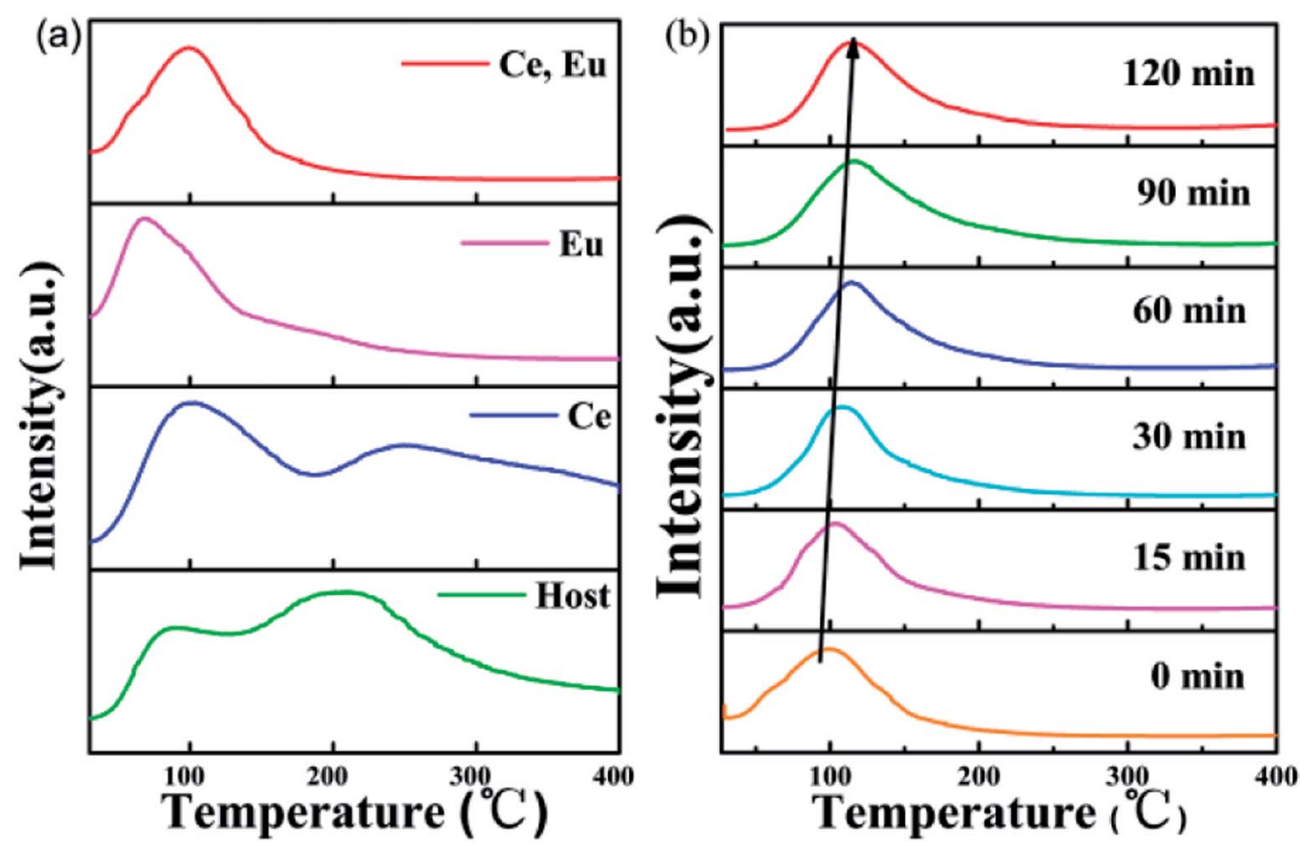

Fig. 7 (a) TL curves of the CAPS, CAPS:0.03C $\mathrm{e}^{3+}, \mathrm{CAPS}: 0.03 \mathrm{Eu}$, and CAPS:0.03Ce, $0.005 \mathrm{Eu}$ samples after irradiation by a UV lamp for 5 min. (b) TL curves of CAPS: $0.03 \mathrm{Ce}^{3+}, 0.005 E u$ at different delay times after irradiation by a UV lamp for $5 \mathrm{~min}$. 
sample CAPS:0.03Eu, the shapes of the TL curves greatly change. The second peak at about $250{ }^{\circ} \mathrm{C}$ almost disappears. When Ce and Eu ions are co-doped, the curve is similar to that of the Eu single sample, except that a weak shoulder peak reappears above $150{ }^{\circ} \mathrm{C}$. These results indicate that $\mathrm{Eu}$ ions are the dominant factor affecting the trap energy and density distribution. Fig. 7(b) and S3(a) $\dagger$ show the TL curves of CAPS:0.03Ce, 0.005Eu and CAPS:0.03Eu at different delay times. As the delay time increases, the TL intensity gradually decreases and the highest peak of the TL curve continuously shifts to a higher temperature; this indicates that the escape rate of the charges captured in shallow traps is much faster than that of those in deep traps at room temperature. ${ }^{37}$

According to the Eeckhout method, ${ }^{38}$ a series of excitation temperature-dependent TL experiments of CAPS:0.03Ce, 0.005Eu and CAPS:0.03Eu were performed, as shown in Fig. 8(a) and $\mathrm{S} 3(\mathrm{~b}) . \dagger$ First, the two samples were exposed to re-excitation by a UV lamp for $5 \mathrm{~min}$ and preheated to a certain temperature ( $T_{\text {exc }}$ ) for $10 \mathrm{~min}$ in a muffle furnace; then, they were cooled to ambient temperature. Thermal disturbance can affect the distribution of trapped electrons by detrapping a portion of the electrons in the shallow traps; therefore, the TL curves measured at different $T_{\text {exc }}$ values provide important information regarding the different unemptied fractions of trapped electrons. Fig. 8(a) and S3(b) $\dagger$ manifest that with increasing $T_{\text {exc }}$, not only does the TL intensity decrease but the maximum TL peak gradually shifts to a higher temperature. Because the trap depth is generally proportional to the temperature of the TL peak, the shift of the TL peak shows that the trap distribution in co-doped CAPS is consecutive and broad. As the $T_{\text {exc }}$ increases, the electrons in the shallow traps are released gradually, and the decrease corresponds to a decrease of the electron density in the traps at a certain depth. The trap depths of these defects can be calculated by the following equation: ${ }^{39}$

$$
E_{\mathrm{T}}=\frac{T_{\mathrm{M}}}{500}
$$

where $E_{\mathrm{T}}$ is the thermo-active energy of the trap depths (in $\mathrm{eV}$ ), denoting the energy gap between the trap levels and the conduction band of the host, and $T_{\mathrm{M}}$ is the temperature of the TL peak (in Kelvin, K). The trap depths of CAPS:0.03Ce, 0.005Eu and CAPS:0.03Eu were calculated to be $0.744,0.818,0.836$, $0.882,0.966,1.028,1.054$, and $1.156 \mathrm{eV}$ and $0.682,0.776,0.824$, $0.868,0.892,0.926,1.022$, and $1.084 \mathrm{eV}$ for the temperatures 298, 323, 348, 373, 398, 423, 448 and $473 \mathrm{~K}$, respectively. Based on this, the trap density and energy distributions can be depicted, as shown in Fig. 8(b) and S3(c). $\uparrow$ These results indicate that traps with different energy depths and density distributions indeed exist in our materials.

Based on the configuration coordinate model, Fig. 9 carefully depicts the possible ET processes among the trap levels, $\mathrm{Ce}^{3+}, \mathrm{Eu}^{2+}$ and $\mathrm{Eu}^{3+}$. With increasing temperature, the traps with appropriate energy depths and density distributions will affect our PL spectra in three probable ways: (1) the radiative transitions from recombination of electron-hole pairs can transfer energy to activators along path (1); (2) direct cross transfer occurs between the trap levels and activator excited states along path (2); (3) the traps near the conduction band capture electrons, release them by thermal excitation to the conduction band, and then compensate for the activator excited states along path (3). In the material CAPS, we do not observe an obvious self-luminescence phenomenon, indicating that the influence of traps on Ce or Eu from path (1) is weak. Efficient ET from the traps to $\mathrm{Ce}$ and $\mathrm{Eu}$ by path (2) requires at least three factors: (1) the energies of the trap levels and the $\mathrm{Ce}$ or Eu excited states must be similar; (2) the numbers of traps with similar energies must be sufficiently high; (3) the distances between traps with similar energies and Ce or Eu must be sufficiently short. We consider that few traps can satisfy these strict conditions in our materials. Thus, the influence of the traps on $\mathrm{Ce}$ or $\mathrm{Eu}$ from path (2) can be neglected. With increasing temperature, the electrons in the traps can leap into the conduction band by a thermal disturbance effect and become free electrons. This is because $\mathrm{Ce}^{3+}$
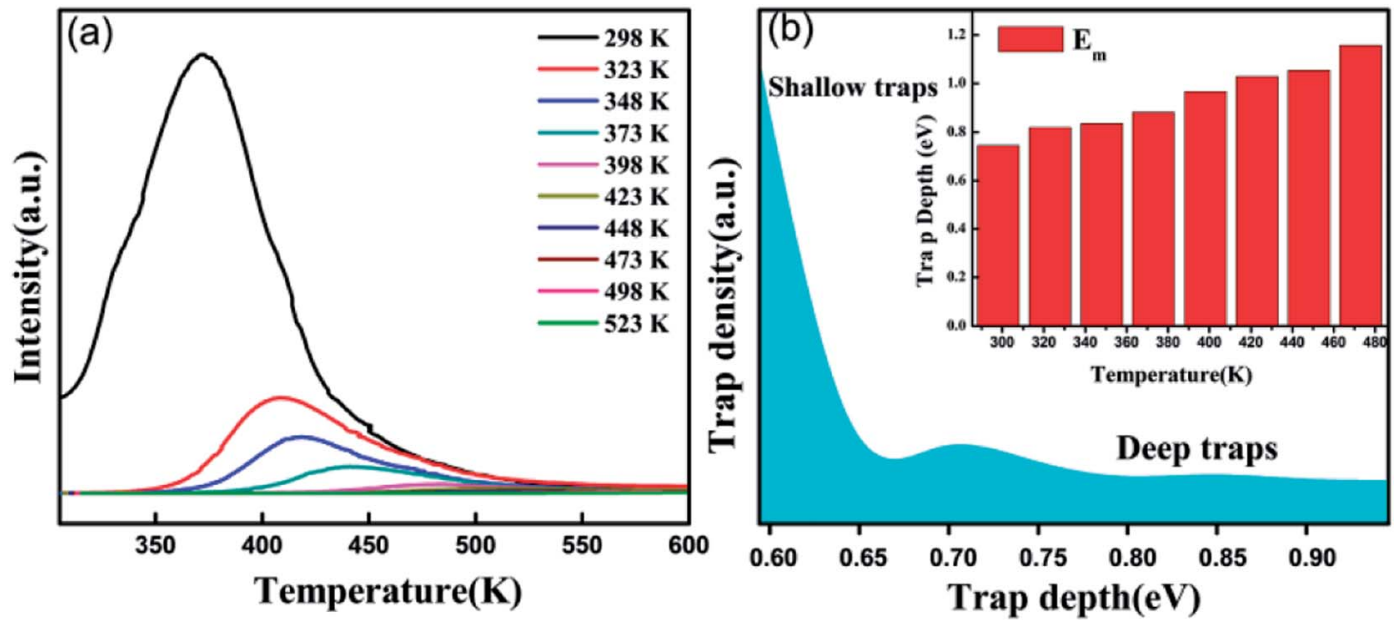

Fig. 8 (a) Excitation temperature-dependent TL curves of CAPS:0.03Ce, $0.005 \mathrm{Eu}$ by pre-exciting for 5 min and pre-heating for 10 min at different $T_{\text {exc. }}$ (b) Trap density and depth distributions in CAPS:0.03Ce, 0.005Eu. 


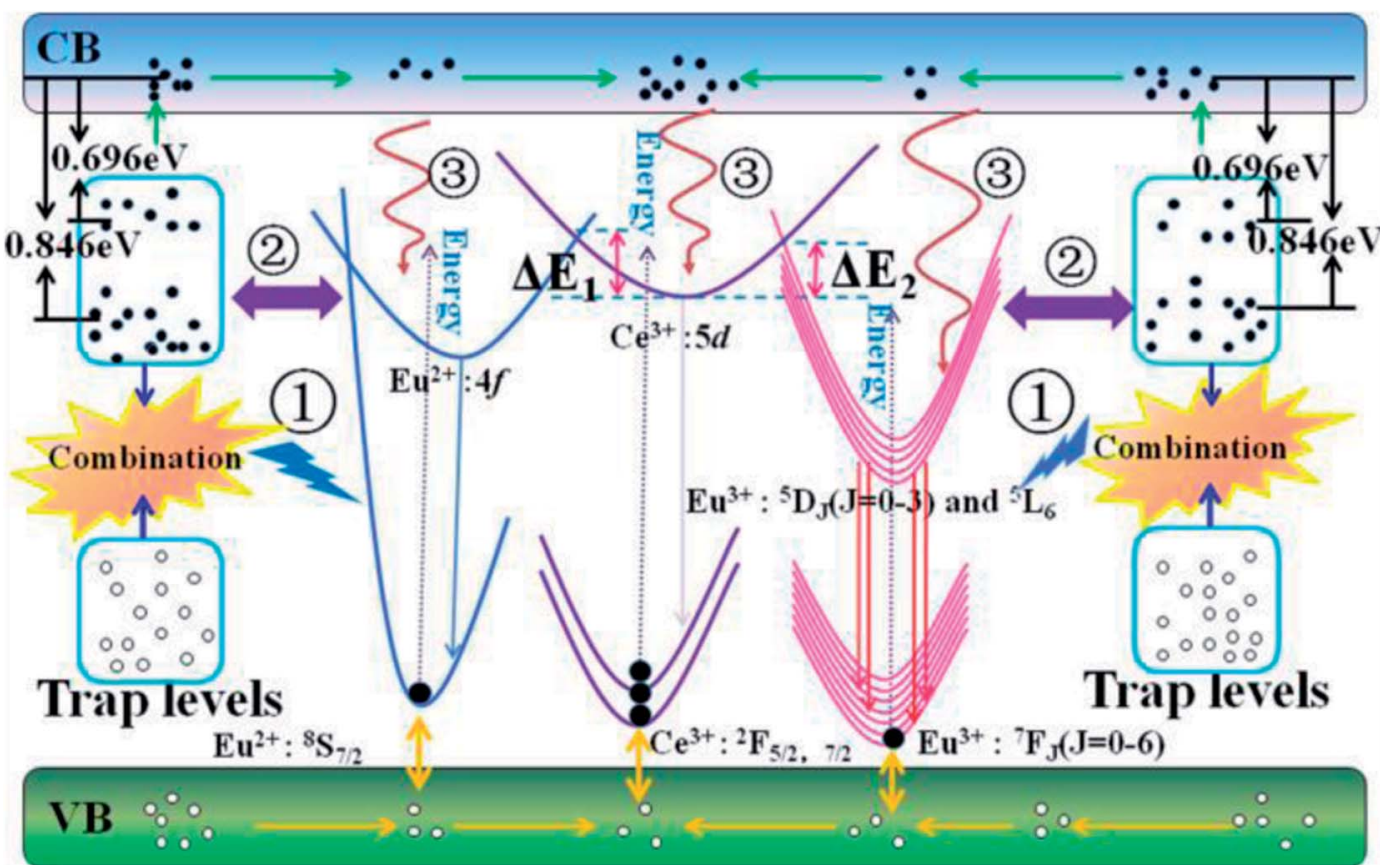

Fig. 9 The configurational coordinate diagram of the excited and ground states of the trap levels, $\mathrm{Ce}^{3+}, \mathrm{Eu}^{2+}$ and $\mathrm{Eu}^{3+}$.

has a higher excitation state and a closer conduction band than $\mathrm{Eu}^{2+}$ and $\mathrm{Eu}^{3+}$. Thus, the vast majority of electrons can relax to the excitation state of $\mathrm{Ce}^{3+}$, and only a few electrons can relax to the excitation states of $\mathrm{Eu}^{2+}$ and $\mathrm{Eu}^{3+}$. As a result, $\mathrm{Ce}^{3+}$ has excellent thermal stability in both the single and codoped systems. For the single and co-doped systems of $\mathrm{Eu}^{2+}$ and $\mathrm{Eu}^{3+}$, it is obvious that the co-doped systems of $\mathrm{Eu}^{2+}$ and $\mathrm{Eu}^{3+}$ present an improvement in thermal stability compared to the single systems of $\mathrm{Eu}^{2+}$ and $\mathrm{Eu}^{3+}$. We can give a reasonable explanation that under the excitation of UV light, the electrons of $\mathrm{Ce}^{3+}$ are excited to the excited states. Then, most of the electrons return to their ground states by radiative transition to obtain the characteristic emission of $\mathrm{Ce}^{3+}$ at room temperature. However, for the Ce and Eu ions co-doped system, the excited state electrons of $\mathrm{Ce}^{3+}$ likely overcome the energy barrier $\Delta E_{1}$ or $\Delta E_{2}$, transferring to the excited states of $\mathrm{Eu}^{2+}$ and $\mathrm{Eu}^{3+}$ under electron-phonon coupling; that is, $\mathrm{Ce}^{3+}$ compensates for the excited state electrons of $\mathrm{Eu}^{2+}$ and $\mathrm{Eu}^{3+}$. Therefore, the decreasing rates of the PL intensities for $\mathrm{Eu}^{2+}$ and $\mathrm{Eu}^{3+}$ in CAPS:0.03Ce, 0.005Eu are suppressed, and the trend even appears to increase.

The temperature-dependent luminescence properties of the typical samples imply that this material can be applied in temperature fluorescence sensors based on the luminescence intensity ratio (FIR). ${ }^{40,41}$ The histogram $\left(I_{614} / I_{339}\right)$ of $\mathrm{Eu}^{3+} / \mathrm{Ce}^{3+}$ in CAPS:0.03Ce, 0.005Eu and the histogram $\left(I_{614} / I_{451}\right)$ of $\mathrm{Eu}^{3+} / \mathrm{Eu}^{2+}$ in CAPS:0.03Eu are presented in Fig. 10(a) and S4(a). $\dagger$ Photos of typical samples are shown in Fig. 10(b) and S4(b). $\dagger$ It can be clearly observed that the luminescence color obviously changes with increasing temperature from $25^{\circ} \mathrm{C}$ to $250^{\circ} \mathrm{C}$. According to Struck and Fonger's theory, ${ }^{42}$ the relationship between the temperature and PL intensity can be expressed as:

$$
\frac{I(T)}{I_{0}}=\left[1+A \exp \left(-\frac{E}{k_{\mathrm{B}} T}\right)\right]^{-1}
$$

where $I_{0}$ is the PL intensity at $0 \mathrm{~K}, A$ is a pre-exponential constant, $k_{\mathrm{B}}$ is the Boltzmann constant, $T$ is the absolute temperature, and $E$ is the thermal quenching activation energy. Based on eqn (7), the FIR of $\mathrm{Eu}^{3+}$ to $\mathrm{Ce}^{3+}\left(I_{614} / I_{339}\right)$ and $\mathrm{Eu}^{3+}$ to $\mathrm{Eu}^{2+}\left(I_{614} / I_{451}\right)$ can be deduced and expressed as follows:

$$
\begin{gathered}
\mathrm{FIR}=\frac{I_{\mathrm{Eu}^{3+}}}{I_{\mathrm{Ce}^{3+}}}=\frac{I_{0, \mathrm{Eu}^{3+}}}{I_{0, \mathrm{Ce}^{3+}}} \frac{1+A_{\mathrm{Ce}^{3+}} \exp \left(-\frac{E_{\mathrm{Ce}^{3+}}}{k_{\mathrm{B}} T}\right)}{1+A_{\mathrm{Eu}^{3+}} \exp \left(-\frac{E_{\mathrm{Eu}^{3+}}}{k_{\mathrm{B}} T}\right)} \\
\approx B+C \exp \left(-\Delta E / K_{\mathrm{B}} T\right)
\end{gathered}
$$

where $A, B, C$ and $\Delta E$ are basic constants for specific hosts. The absolute and relative temperature sensitivities, $S_{\mathrm{a}}$ and $S_{\mathrm{r}}$, can be further derived and expressed by the following equations:

$$
\begin{aligned}
S_{\mathrm{a}} & =\left|\frac{\partial \mathrm{FIR}}{\partial T}\right|=C \exp \left(-\Delta E / K_{\mathrm{B}} T\right) \times \frac{\Delta E}{k_{\mathrm{B}} T^{2}} \\
S_{\mathrm{r}} & =100 \% \times\left|\frac{1}{\mathrm{FIR}} \frac{\partial \mathrm{FIR}}{\partial T}\right| \\
& =100 \% \times \frac{C \exp \left(-\frac{\Delta E}{K_{\mathrm{B}} T}\right)}{B+C \exp \left(-\frac{\Delta E}{K_{\mathrm{B}} T}\right)} \times \frac{\Delta E}{K_{\mathrm{B}} T^{2}}
\end{aligned}
$$

As displayed in Fig. 10(c) and S4(c), $\dagger$ the measured plots of FIR versus temperature can be fitted well by eqn (8). The $S_{\mathrm{a}}$ and $S_{\mathrm{r}}$ values calculated by eqn (9) and (10) are presented in Fig. 10(d) and S4(d). $\dagger$ Detailed data for $S_{\mathrm{a}}$ and $S_{\mathrm{r}}$ are listed in 

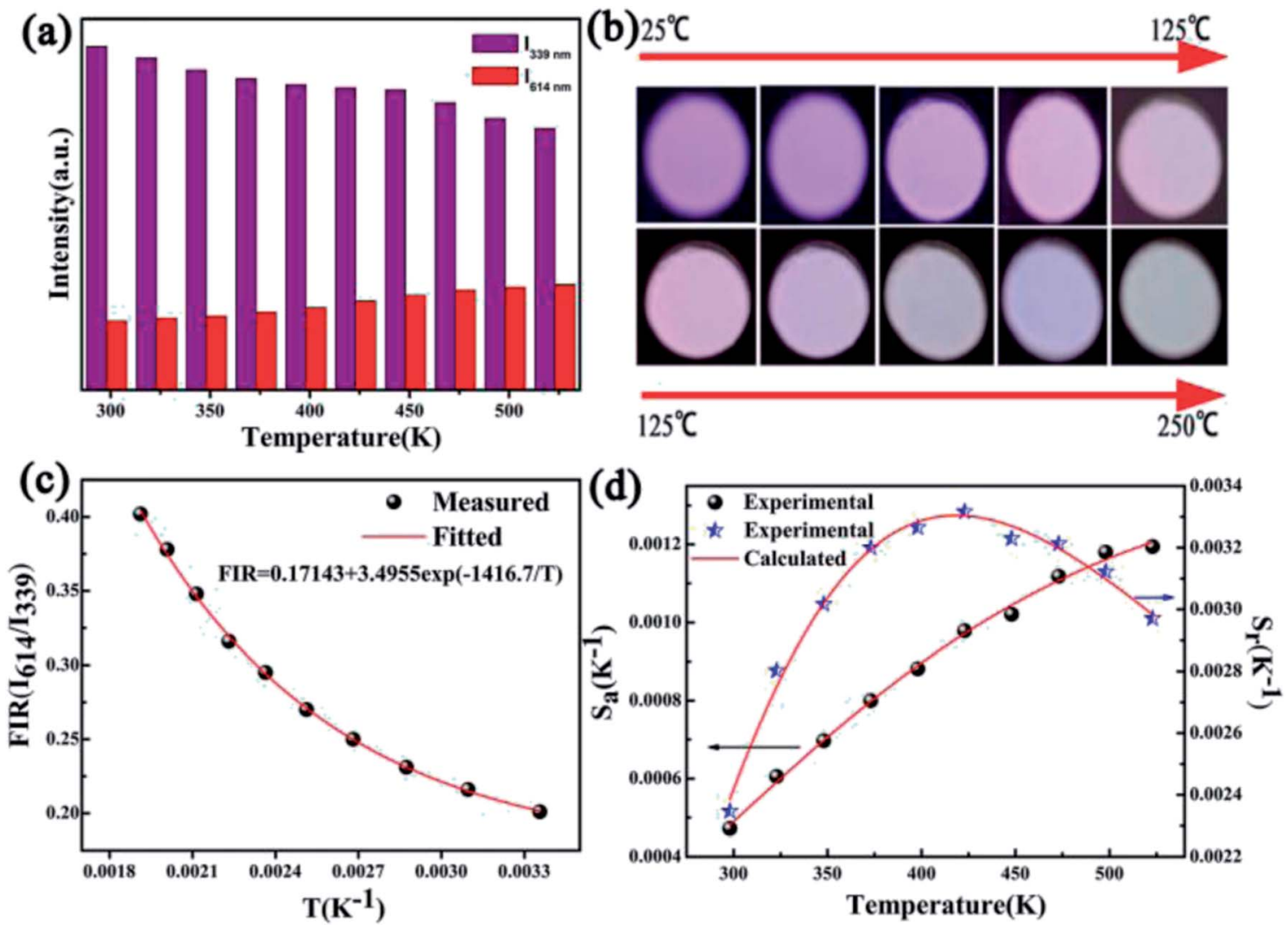

Fig. 10 (a) Histogram displaying the luminescence intensities of $\mathrm{Eu}^{3+}(\approx 614 \mathrm{~nm})$ and $\mathrm{Ce}^{3+}(\approx 339 \mathrm{~nm})$ at various temperatures. (b) Photos of the sample at various temperatures under UV irradiation. (c) Experimentally measured and eqn (8)-fitted plots of FIR $\left(I_{614} / I_{339}\right)$ versus temperature. (d) Absolute sensitivity $S_{a}$ and relative sensitivity $S_{r}$ versus temperature.

Tables S1 and S2.† Although the CAPS:0.03Eu sample has a greater absolute temperature sensitivity $S_{\mathrm{a}}$, in fact, Fig. 10(c) presents better monotonicity than Fig. S4(c). $\uparrow$ The photos in Fig. 10(b) and S4(b) $\dagger$ also obviously show that the luminescence color becomes indistinguishable when the temperature reaches $125^{\circ} \mathrm{C}$ for the sample CAPS:0.03Eu. Thus, we consider that the Ce and Eu co-doped CAPS samples have better signal discriminabilities than Eu single CAPS in a broad temperature range, which is beneficial for application in fluorescent temperature sensors.

\subsection{CL properties}

To more profoundly investigate the physical process of electron transition upon excitation from different sources and explore their potential application in FEDs, the CL spectral properties of CAPS:0.03Ce, $x \mathrm{Eu}(0.001 \leq x \leq 0.07)$ were investigated and revealed typical emissions of $\mathrm{Ce}^{3+}, \mathrm{Eu}^{2+}$, and $\mathrm{Eu}^{3+}$; the results are shown in Fig. S5(a). $\uparrow$ The CIE chromaticity diagram and coordinates for the CAPS:0.03Ce, $x$ Eu phosphors are shown in Fig. S5(b) and Table S3. $\dagger$ Compared with the PL spectra under excitation at 265 and $296 \mathrm{~nm}$, the intensity ratio of $I_{441} / I_{339}$ of the CL spectrum for the sample CAPS:0.03Ce, 0.005Eu was greatly strengthened while the intensity ratio of $I_{614} / I_{339}$ showed no remarkable change, as shown in Fig. 11(a) and (b). From the energy level diagram in Fig. 11(d), the phenomenon can be reasonably explained as follows. For the PL spectrum, the excitation sources of 265 and $296 \mathrm{~nm}$ only provide energies of 3 to $4 \mathrm{eV}$, which can excite partial electrons of the energy levels of $\mathrm{Ce}^{3+}, \mathrm{Eu}^{2+}, \mathrm{Eu}^{3+}$ and the traps. Meanwhile, an electron beam can provide energies of several thousand and even tens of thousands of eV. The high-energy particles bombard the sample and bring out more secondary electrons with decreasing electron energy. Finally, a large number of electrons in the valence band will be excited to the conduction band, and some of these may excite or transfer energy to the activators and traps. Most of the excited electrons in the conduction band can move and gradually aggregate in the excited state of $\mathrm{Ce}^{3+}$ and the trap levels, as shown in Fig. 11(d). In Fig. 5, we show that the decay time of $\mathrm{Ce}^{3+}$ is on the order of nanoseconds. The electrons in the excited states of $\mathrm{Ce}^{3+}$ can transfer quickly to the ground state by radiative transition. Because the cardinal number of electrons in the excited states is large, electron saturation of the $\mathrm{Ce}^{3+}$ ground state is very likely. The redundant electrons in the $\mathrm{Ce}^{3+}$ excited state can be easily transferred to the excited states of $\mathrm{Eu}^{2+}$ and $\mathrm{Eu}^{3+}$ through ET, correspondingly increasing the $\mathrm{Eu}^{2+}$ and $\mathrm{Eu}^{3+}$ emission intensities. As we know, the decay times of $\mathrm{Eu}^{2+}$ and $\mathrm{Eu}^{3+}$ are usually on the order of microseconds and milliseconds. ${ }^{43}$ The electrons in the $\mathrm{Eu}^{2+}$ and $\mathrm{Eu}^{3+}$ ground states have enough time to return to the valence band, suppressing the occurrence of ground state saturation. Also, because the radiation rate of $\mathrm{Eu}^{2+}$ is faster than that of $\mathrm{Eu}^{3+}$, the $\mathrm{Eu}^{2+}$ 
(a)

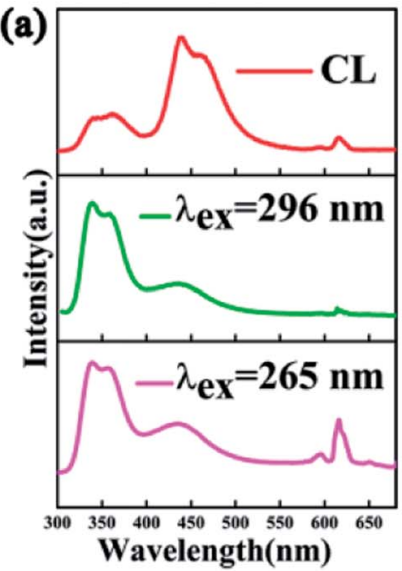

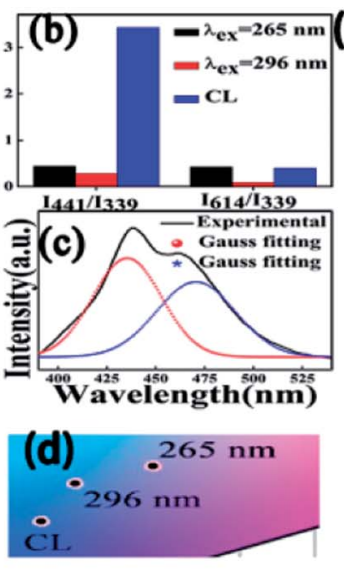

(e) $\mathrm{CB}$

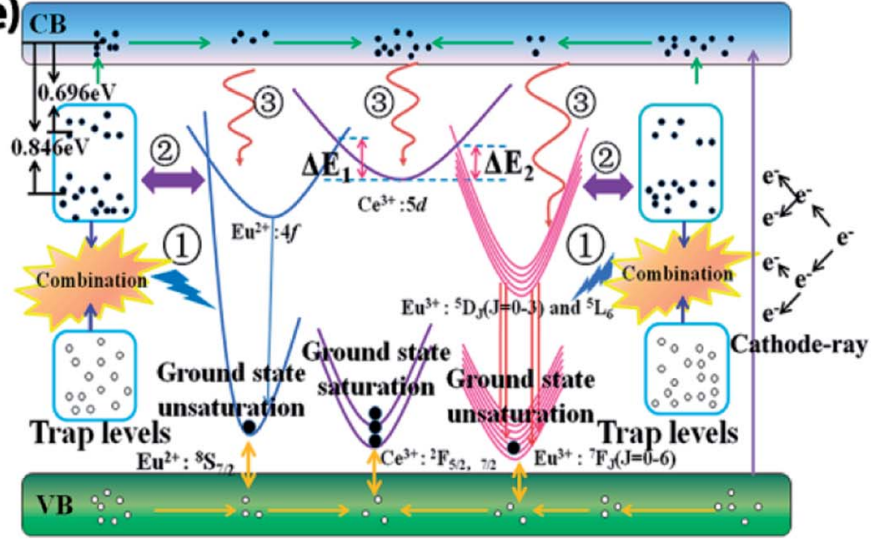

Accelerating voltage(KV)

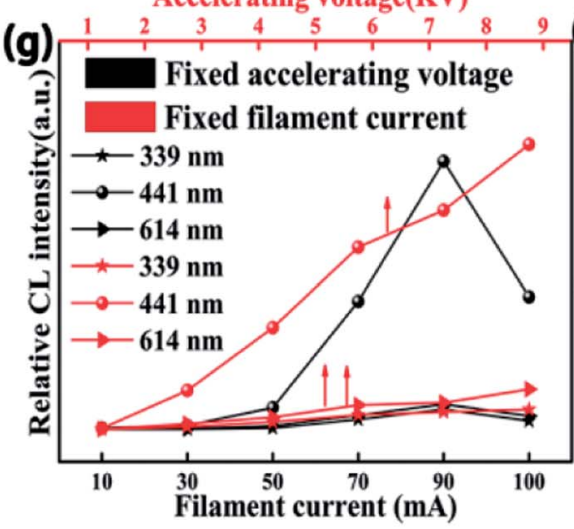

Accelerating voltage(KV)

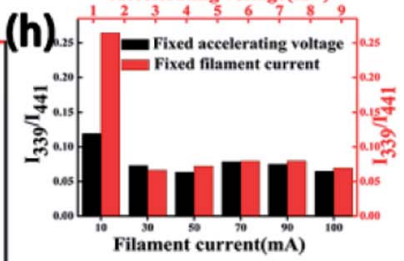

(i)

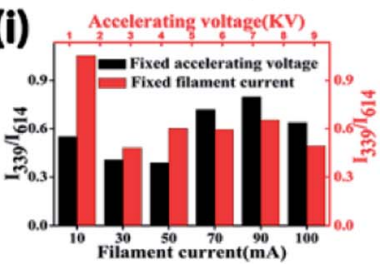

Fig. 11 (a) PL curves excited at 296 and $265 \mathrm{~nm}$ and CL curves at an accelerated voltage of $5 \mathrm{kV}$ and a filament current of $70 \mathrm{~mA}$ for CAPS:0.03Ce, $0.005 \mathrm{Eu}$. (b) The intensity ratios of $I_{441} / I_{339}$ and $I_{614} / I_{339}$ upon excitation from different sources. (c) The Gauss curves of the PL spectra of CAPS:0.03Ce, 0.005Eu. (d) The CIE chromaticity coordinates of CAPS:0.03Ce, 0.005Eu upon excitation from different sources. (e) Schematic energy level diagram. (f) The intensity of degradation of CAPS:0.03Ce, $0.005 \mathrm{Eu}$ at different times. (g) The relative CL intensities of CAPS:0.03Ce, $0.005 \mathrm{Eu}$ at different accelerating voltages and filament currents. (h) and (i) intensity ratios of $I_{339} / I_{441}$ and $I_{339} / I_{614}$.

emission finally presents a more obvious change than the $\mathrm{Ce}^{3+}$ and $\mathrm{Eu}^{3+}$ emissions. In addition, in the above content, we have discussed possible three paths (1), (2) and (3) of trap influence on the activators. Under excitation by electron beams, the electrons in the conduction band are much more numerous than under excitation by UV light because of the extremely high energy of the excitation source. This may be the reason why the contributions of the traps in the CL spectra are relatively smaller than those in the PL spectra. Fig. 11(c) shows the $\mathrm{Eu}^{2+}$ emission band from 380 to $525 \mathrm{~nm}$. Two distinct peaks at 435 and $475 \mathrm{~nm}$ can be observed by Gauss fitting, which is completely coincident with the PL spectrum in Fig. 3 and further indicates that $\mathrm{Eu}^{2+}$ occupies two different sites with $8^{-}$and 9-coordination. Fig. 11(d) presents the CIE chromaticity coordinates of CAPS:0.03Ce, 0.005Eu upon excitation from different sources. The degradation behaviour of CAPS:0.03Ce, $0.005 \mathrm{Eu}$ under a continuous fixed accelerating voltage $(5 \mathrm{kV})$ and a fixed filament current (70 mA) was measured and is shown in Fig. 11(f). It is apparent that the CL intensities of three peaks $(339,441$ and $614 \mathrm{~nm}$ ) slowly decrease with increasing bombing time, which may be due to the following four reasons: (1) accumulation of graphitic carbon on the surface of the samples during continuous electron bombardment, resulting in carbon contamination, (2) accumulation of surface charges, (3) thermal quenching effects caused by the increase of the surface temperature of the sample over the long period of electron beam bombardment, (4) ground-state depletion. ${ }^{44-47}$ In our work, the sample CAPS:0.03Ce, 0.005Eu has good degradation properties. When bombarded by a persistent electron beam for $90 \mathrm{~min}$, the $\mathrm{CL}$ intensities of $\mathrm{Ce}^{3+}, \mathrm{Eu}^{2+}$, and $\mathrm{Eu}^{3+}$ decreased to $67.1 \%, 72.3 \%$ and $71.8 \%$ of the initial values, respectively. Fig. $11(\mathrm{~g})$ shows the CL intensities of the CAPS:0.03Ce, $0.005 \mathrm{Eu}$ sample as a function of the accelerating voltage and filament current. With increasing voltage from $1 \mathrm{kV}$ to $6 \mathrm{kV}$ with a fixed current at $70 \mathrm{~mA}$, the electronic penetration depth increased continually; more luminescence centers will be activated and release energy, resulting in this continuous enhancement in the emission intensity. When increasing the filament current from $10 \mathrm{~mA}$ to $100 \mathrm{~mA}$ under $5 \mathrm{KV}$ accelerating voltage, the same penetration layer of our sample has a larger electron density, which will also cause more luminescence centers to be activated; thus, the CL emission intensities also gradually increase and reach a maximum at $90 \mathrm{~mA}$ due to ground-state depletion, the thermal quenching effect and charge buildup effects. It is 
noteworthy that the CL intensity at variable voltage increases more rapidly than that at variable current, which implies that the influence of the penetration depth is greater than that of the electron density. In addition, the intensity ratios of both $I_{339} / I_{441}$ and $I_{339} / I_{614}$ gradually changed and stabilized with increasing accelerating voltage and filament current.

\section{Conclusion}

Temperature-dependent fluorescence is a key index of rare earth ion-doped functional materials. In this work, to obtain luminous-self-healing properties, we successfully synthesized a multi-cationic site phosphosilicate phosphor, $\mathrm{Ca}_{8} \mathrm{Al}_{2} \mathrm{P}_{6}$ $\mathrm{SiO}_{28}$ :Ce, Eu. By XRD, PL and PLE spectra, decay times, CIE chromaticity coordinates, thermal quenching, etc., the temperature-dependent fluorescence properties and effects of traps on the emissions of the activators were investigated in detail. Under UV light excitation, $\mathrm{Ca}_{8} \mathrm{Al}_{2} \mathrm{P}_{6} \mathrm{SiO}_{28}$ : Ce showed a broad blue-violet band due to the $\mathrm{Ce}^{3+} \mathrm{d}-\mathrm{f}$ transition. Meanwhile, for the $\mathrm{Ca}_{8} \mathrm{Al}_{2} \mathrm{P}_{6} \mathrm{SiO}_{28}$ :Eu sample, Eu could not be completely reduced and was present in coexisting states of $\mathrm{Eu}^{2+}$ and $\mathrm{Eu}^{3+}$. By designing the synthesis schemes of the target materials, the energy depths and density distributions of the traps could be reasonably adjusted. Finally, we realized selfsuppression of the emission loss by energy compensation from traps or ET between Ce and Eu ions in the material CAPS. With increasing temperature, for the typical single and codoped samples, the luminescence intensities of $\mathrm{Ce}^{3+}$ at $250{ }^{\circ} \mathrm{C}$ were $81 \%$ and $76 \%$ of their initial intensities at ambient temperature, which may be due to energy compensation from traps; the luminescence intensities of $\mathrm{Eu}^{2+} / \mathrm{Eu}^{3+}$ at $250{ }^{\circ} \mathrm{C}$ were $10 \% / 47 \%$ and $58 \% / 52 \%$, respectively, which mainly resulted from ET between $\mathrm{Ce}$ and $\mathrm{Eu}^{2+} / \mathrm{Eu}^{3+}$. This phenomenon implies that CAPS can be applied in fluorescent temperature sensors. Upon excitation by electron beams, the shapes of the emission spectra were obviously different from those of the PL spectra. Based on the CL curves at an accelerated voltage of $5 \mathrm{KV}$ and a filament current of $70 \mathrm{~mA}$, the degradation intensities at different times and the CL intensity ratios at different accelerating voltages and filament currents, we carefully illuminated the photo-physical mechanism of electron transition by a configurational coordinate diagram. These results can be useful in the discussion of trap energy compensation or ET effects for luminous-self-healing materials or abnormal luminescence changes with different excitation sources.

\section{Conflicts of interest}

There are no conflicts to declare.

\section{Acknowledgements}

This work was supported by the National Natural Science Funds of China (no. 51302121 and 51462031), the Natural Science Foundation of Gansu Province of China (Grant No. 1606RJYA262), the Scientific research projects of Gansu colleges and Universities (Grant No. 2017A-009), China Postdoctoral
Science Foundation (Grant No. 2016M592909XB), Creation of Science and Technology of Northwest Normal University, China (Grant No. NWNU-LKQN-15-9) and the Fundamental Research Funds for the Central Universities (lzujbky-2017-sp23).

\section{References}

1 J. C. Bünzli and C. Piguet, Chem. Soc. Rev., 2006, 37, 10481077.

2 K. Binnemans, Chem. Rev., 2009, 109, 4283-4374.

3 X. D. Wang, O. S. Wolfbeis and R. J. Meier, Chem. Soc. Rev., 2013, 42, 7834-7869.

4 V. Bachmann, C. Ronda and A. Meijerink, Chem. Mater., 2009, 21, 2077-2084.

5 T. Wang, J. Gou and X. Xu, Opt. Express, 2015, 23, 1259512604.

6 G. Wang, Q. Peng and Y. Li, J. Am. Chem. Soc., 2009, 131, 14200-14201.

7 J. Zhao, D. Jin, E. P. Schartner, Y. Lu, Y. Liu and A. V. Zvyagin, Nat. Nanotechnol., 2013, 8, 729-734.

8 L. Wang and Y. Li, Nano Lett., 2006, 6, 1645.

9 J. K. Cao, X. M. Li, Z. X. Wang, Y. L. Wei, L. P. Chen and H. Guo, Sens. Actuators, B, 2016, 224, 507-513.

10 J. K. Cao, D. K. Xu, F. F. Hu, X. M. Li, W. P. Chen, L. P. Chen and H. Guo, J. Eur. Ceram. Soc., 2018, 38, 2753-2758.

11 A. Pandey, V. K. Rai, V. Kumar and H. C. Swart, Sens. Actuators, B, 2015, 209, 352.

12 X. Huang, S. Han, W. Huang and X. Liu, Chem. Soc. Rev., 2013, 42, 173-201.

13 J. Xu, S. Tanabe, A. D. Sontakke and J. Ueda, Appl. Phys. Lett., 2015, 107, 58674.

14 Y. H. Kim, P. Arunkumar and Y. K. Bo, Nat. Mater., 2017, 16, 543.

15 K. Li, D. Geng, M. Shang, Y. Zhang, H. Lian and J. Lin, J. Phys. Chem. C, 2014, 118, 11026-11034.

16 J. K. Cao, W. P. Chen, D. K. Xu, F. F. Hu, L. P. Chen and H. Guo, J. Lumin., 2018, 194, 219-224.

17 J. K. Cao, F. F. Hu, L. P. Chen, H. Guo, C. K. Duan and M. Yin, J. Am. Ceram. Soc., 2017, 100, 2108-2115.

18 P. Dorenbos, J. Phys. Condens. Matter, 2005, 17, 8103-8111.

19 Y. H. Kim, P. Arunkumar, B. Y. Kim, S. Kim, E. Unithrattil and S. H. Moon, Nat. Mater., 2017, 16, 543.

20 R. D. Shannon, Acta Crystallogr., Sect. A: Cryst. Phys., Diffr., Theor. Gen. Crystallogr., 1976, 32, 751-767.

21 B. I. Lazoryak, T. V. Strunenkova, E. A. Vovk and V. V. Mikhailin, Mater. Res. Bull., 1996, 31, 665-671.

22 L. G. V. Uitert, J. Lumin., 1984, 29, 1-9.

23 Z. W. Zhang, H. Zhong, S. S. Yang and X. J. Chu, J. Alloys Compd., 2017, 708, 671-677.

24 A. M. Pires, M. R. Davolos and O. L. Malta, J. Lumin., 1997, 72, 244-246.

25 J. Yang, L. Yang, W. Liu, Y. Zhang, H. Fan and Y. Wang, J. Alloys Compd., 2008, 454, 506-509.

26 B. Piriou, M. Richard-Plouet, J. Parmentier, F. Ferey and S. Vilminot, J. Alloys Compd., 1997, 262, 450-453.

27 M. Buijs, A. Meyerink and G. Blasse, J. Lumin., 1987, 37, 9-20. 28 C. K. Chang and T. Chen, Appl. Phys. Lett., 2007, 91, 46. 
29 K. A. Denault, J. Brgoch, M. W. Gaultois, A. Mikhailovsky, R. Petry and H. Winkler, Chem. Mater., 2014, 26, 2275.

30 M. Li, X. Yu, X. Xu, S. Cheng, B. Zhang and J. Qiu, J. Am. Ceram. Soc., 2015, 98, 2008-2010.

31 F. Kang, Y. Zhang and M. Peng, Inorg. Chem., 2015, 54, 14621473.

32 Y. Su, L. Li and G. Li, J. Cheminf., 2008, 20, 6060-6067.

33 M. Yu, J. Lin and J. Fang, Chem. Mater., 2005, 17, 1783-1791.

34 Z. Xia, Y. Zhang, M. S. Molokeev and V. V. Atuchin, J. Phys. Chem. C, 2013, 117, 20847-20854.

35 P. Dorenbos, J. Phys. Condens. Matter, 2005, 17, 8103-8111.

36 J. Zhou, J. Zhong, J. Guo, H. Liang, Q. Su and Q. Tang, J. Phys. Chem. C, 2016, 120, 18741-18747.

37 X. Li, X. Tang, Z. Wang, Z. Zou, J. Zhang and Z. Ci, J. Alloys Compd., 2017, 721, 512-519.

38 K. V. D. Eeckhout, A. J. J. Bos, D. Poelman and P. F. Smet, Phys. Rev. B, 2013, 87, 045126.
39 Y. H. Kim, P. Arunkumar, B. Y. Kim, S. Unithrattil, E. Kim, S.-H. Moon, J. Y. Hyun, K. H. Kim, D. Lee, J.-S. Lee and W. B. Im, Nat. Mater., 2017, 16, 543-550.

40 T. V. Gavrilovic, D. J. Jovanovic, V. Lojpur and M. D. Dramicanin, Sci. Rep., 2014, 4, 4209; C. W. Struck and W. H. Fonger, J. Appl. Phys., 1971, 42, 4515.

41 A. Pandeya, V. K. Rai, V. Kumar, V. Kumar and H. C. Swart, Sens. Actuators, B, 2015, 209, 352-358.

42 C. W. Struck and W. H. Fonger, J. Appl. Phys., 1971, 42, 4515.

43 Z. Xia, S. Miao, M. Chen, M. S. Molokeev and Q. Liu, Inorg. Chem., 2015, 54, 7684-7691.

44 F. L. Zhang, S. Yang, C. Stoffers, J. Penczek and P. N. Yocom, Appl. Phys. Lett., 1998, 72, 2226-2228.

45 M. Shang, G. Li, D. Yang, X. Kang, C. Peng, Z. Cheng and J. Lin, Dalton Trans., 2011, 40, 9379-9387.

46 Q. Sun, X. Li and Y. Du, J. Am. Ceram. Soc., 2017, 100, 193203.

47 N. Zhang, C. Guo and H. Jing, RSC Adv., 2013, 3, 7495-7502. 